\title{
PET/CT and Hypo-Fractionated Radiotherapy of Patients with Head and Neck Cancer
}

\author{
Misleidy Nápoles Morales', Aldo Martínez Ramirez², Juan P. Oliva Glez², \\ Claudia González Espinosa², Joaquín González González², Carlos Fabián Calderón Marín², \\ Waldo Quesada Cepero², Fanny López Reina1, Rubén Elzaurdín Mora ${ }^{3}$ \\ ${ }^{1}$ Department of Radiotherapy, Institute of Oncology and Radiobiology, Havana, Cuba \\ ${ }^{2}$ Nuclear Medicine Department and Molecular Imaging, Institute of Oncology and Radiobiology, Havana, Cuba \\ ${ }^{3}$ Radiotherapy Department, Medical Researchers Center, Havana, Cuba \\ Email: misleidy.napoles@infomed.sld.cu
}

How to cite this paper: Morales, M.N., Ramirez, A.M., Glez, J.P.O., Espinosa, C.G., González, J.G., Marín, C.F.C., Cepero, W.Q., Reina, F.L. and Mora, R.E. (2018) PET/CT and Hypo-Fractionated Radiotherapy of Patients with Head and Neck Cancer. International Journal of Clinical Medicine, 9, 751-759.

https://doi.org/10.4236/ijcm.2018.910062

Received: September 4, 2018

Accepted: October 22, 2018

Published: October 25, 2018

Copyright $\odot 2018$ by authors and Scientific Research Publishing Inc. This work is licensed under the Creative Commons Attribution International License (CC BY 4.0).

http://creativecommons.org/licenses/by/4.0/

\begin{abstract}
Positron emission tomography/Computer tomography (PET/CT) is a multimodality imaging diagnostic technique that analyzes the uptake and retention of different radiopharmaceuticals by cells providing metabolic information on biochemical processes. PET/CT has been used for radiotherapy planning, providing useful information to the Radio-oncologist about the localization, size and metabolic activity of tumor lesions. In this paper, we show advantages of the 18F-FDG PET/CT respect to simple CT imaging for target volume delineation in patients with diagnosis of Squamous Head and Neck Carcinoma that has been scheduled to undergo a hypofractionated radiotherapy treatment. On ten studied patients, the target volume defined from PET/CT images was less extensive than those defined from simple CT images. In six patients the target volume was significantly less extensive and in two of them a new lymph node disease was reported, re-staging and corresponding target volume was also delineated with less extensive margins from PET/CT images. A greater accuracy in delineating the volumes and improving the distribution of doses in the planning of the radiant treatment in these patients was possible, allowing a high precision in the delivery of the prescribed dose to the target volume diminishing the maximum dose to the adjacent healthy tissues. In conclusion we show that the use of $18 \mathrm{~F}-\mathrm{FDG}$ PET/CT was superior than the simple CT as the primary modality of imaging for hypofractioned radiotherapy treatment planning in patients with Squamous Head and Neck Carcinoma.
\end{abstract}

\section{Keywords}

PET-CT, Hypofractionated Radiotherapy, Head and Neck Cancer 


\section{Introduction}

Squamous cell carcinoma of the head and neck (HNCC) is the sixth most common cancer with an incidence of approximately 600,000 cases per year and 300,000 deaths per year worldwide [1] [2]. In Cuba, cancer of the larynx is the fourth cause in incidence [3]. It preferentially affects male patients older than 50 years and its main risk factors are smoking and excessive alcohol consumption. However, an epidemiological transition is currently observed due to an increased incidence of head and neck cancer associated with human papillomavirus infection. The most common histological type is squamous, covering more than $90 \%$ of cases [1].

The use of different imaging modalities is essential for the diagnosis treatment and follow-up of patients with HNCC. Computed tomography (CT) and magnetic resonance imaging (MRI) are the conventional diagnostic techniques (CDT) that provide a structural image and allow identifying changes in size and anatomical distortion. These images modalities are often used for target volume delineation however, in case of CT images due to low contrast of soft tissues hinders target volume delineation [4].

Positron emission tomography (PET) is a nuclear medicine imaging diagnostic technique that analyzes the uptake and retention of different radiopharmaceuticals by cells and provides metabolic information on biochemical processes. Improve the detection of tumor lymph node infiltration and distant metastasis with respect to CDT. It allows characterizing if the structural alterations are due to the tumor, to sequels of previous treatments or to other biological processes. Therefore, PET provides additional information which complements the CDT [5] [6].

The treatment of these tumors contemplates different alternatives, the main ones being surgery and radiotherapy associated or not with chemotherapy and/or biological therapies, depending on the tumor stage and the functional reserve of the patient [7]. Radiotherapy has a very well established role either in the exclusive treatment or as adjuvant treatment. It offers possible benefits for allowing the cure of the patient, the preservation of organ functionality, and its cost-effectiveness. The evolution of the radiant methods from the beginning conventional radiotherapy looks for to reduce to the minimum the exposition of healthy tissue neighbor to the tumor and to maximize the dose in the tumor [8].

The use PET/CT in HNSCC is increasingly widespread. It has been used for the radiotherapy planning, especially for unconventional treatments such as: hypofractionated radiotherapy, where the treatment time is decreased by increasing the daily dose obtaining a similar tumor response than conventional fractionation in HNSCC [9].

The purpose of this paper is to show advantages of the 18F-FDG PET/CT respect to simple CT imaging for target volume delineation in patients with diagnosis of HNSCC that has been scheduled to undergo a hypofractionated radiotherapy treatment. 


\section{Material and Methods.}

\subsection{PET/CT Image Acquisition for Hypofractionation Radiotherapy Planning of HNSCC}

The ${ }^{18}$ F-FDG images were acquired in a Philips Gemini TF64 PET/CT system at Nuclear Medicine and Molecular Imaging Department belonging to Oncology and Radiobiology Institute (INOR), La Habana, Cuba. The PET component is a 3D tomograph with time-of-flight (TFO) capability. The CT component of the Philips Gemini system is a 64 slices Brilliance tomograph. Each patient received 3.7 MBq/kg body weight of ${ }^{18} \mathrm{~F}-\mathrm{FDG}$ by intravenous injection. After $60 \mathrm{~min}$ post-administration the patient is positioned in supine position and immobilized using an individually customized head mask which is fixed to a rigid head-holder toenhance the positioning accuracy and to prevent movement during image acquisition. The head holder is then fixed to a Philips Gemini PET/CT Overlay plane couch (http://civcort.com). An initial scout view was used to define a region from skull to the clavicles. A helical CT scan was performed over the region defined with the scout view using the parameters $120 \mathrm{kVp}, 120 \mathrm{mAs}$, with pitch around 1, reconstructed slice thickness of $2.5 \mathrm{~mm}$. The PET TOF emission data were acquired for $1.5 \mathrm{~min} / \mathrm{bed}$, with adequate bed number that lets includes with the acquisition FOV at maximum size in order to assure that patient shoulders were included in the image and the patient border could be delimited during the treatment planning. The CT was used for attenuation correction of PET data. The PET, CT slice reconstructions and spatial co-registration PET/CT were done with the Philips Extended Brilliance ${ }^{\mathrm{TM}}$ Workspace (Philips, version 4.5.2.40008).

\section{Patient data}

Paired study was performed on patients who were irradiated with highly conformed techniques such as IMRT and received the dose in a hypofractioned form. A prospective, observational study was carried out in which 10 patients diagnosed in the Oncology and Radiobiology Institute (INOR) with loco-regional squamous cell carcinoma in pharynx, larynx and oral cavity included in the period between January-July 2018.

\section{Inclusion criteria}

1) Tumor classified as stage I-IV located in oropharynx, hypopharynx, larynx (not glottic stage I-II), or oral cavity according to the TNM classification of Malignant Tumors;

2) Histopathological diagnosis of invasive squamous cell carcinoma at the primary site;

3) Age > 18 years;

4) Informed consent according to the Helsinki declaration and local regulations;

5) The patient must be a candidate for external beam radical radiotherapy, and must be expected to complete the treatment;

6) World Health Organization (WHO) performance status of 0 - 2.

\section{Exclusion criteria}


1) Distant metastases;

2) The patient should not be in a state or have major co-morbidity that could be expected to influence the outcome of treatment, or interfere with the assessment of treatment outcome at follow-up, or (apart from the present disease) considerably reduce the life expectancy;

3) Patients who test positive for human immunodeficiency virus (HIV);

4) Prior surgical excision (except biopsy);

5) Planned (elective) surgery;

6) The existence of synchronous multiple malignancies (not leukoplakia) or previous history of cancer;

7) The patient must not be pregnant;

8) Socio-demographic or other factors that make it unlikely that the patient will be available for follow up of long term treatment outcome.

\subsection{Target Delineation and Radiotherapy Planning}

The spatial co-registered PET/CT images were transferred to Monaco radiotherapy planning system (5.11.02). The organs at risk (OAR) were delineated manually on CT images and target volumes (tumors and nodes) were delineated on both PET and CT images following the Gregory's guideline [10]. The sizes of target volume of interest (VoIs) drawn for tumor and node on both image modalities were calculated. The intra-patient absolute relative differences of VoI sizes were calculated.

The patients who fulfill the inclusion criteria described above received a hypofractionated radiotherapy treatment with a daily dose of 2.75 Gy (5 sessions/week) and $55 \mathrm{~Gy}$ as the total dose in the high-risk tumor target volume (CTV-Hi) while the low-risk tumor target volume (CTV-Lo) received a daily dose of $2.2 \mathrm{~Gy}$ ( 5 sessions/week) and $44 \mathrm{~Gy}$ as the total dose. The doses were delivered with the Modulated Intensity Technique (IMRT), performed in the radiotherapy planning system, Elekta Xio (Version 5.10). The treatment was delivered using an Elekta Synergy linac 2950. The patient position is verified weakly with a cone-beam tomographic image system (CBCT) coupled to the linac.

\section{Results}

As shown in Table 1, the most frequent age group of patients included in the study was between 55 - 70 years. All patients were male and $90 \%$ of them with toxic habits (cigar and alcohol) (Table 1).

The most frequent site of the primary tumor was the Oropharynx (80\%) and $90 \%$ of the patients were at the locally advanced stage of the disease at diagnosis III and IVA.

\section{Impact of Target Delineation Using PET/CT Images}

The metabolic images obtained with the use of the 18FDG-PET-CT when being fused with the planning CT for the radiotherapy facilitated the design of the targets 
Table 1. Demographic and clinical patients characteristic.

\begin{tabular}{ccccccc}
\hline Patients & Sex & Age & Toxic Habit & TNM & Stage & Site \\
\hline 1 & Male & 55 & yes & T2 N2b M0 & IVA & tonsil \\
2 & Male & 67 & yes & T2 N3 M0 & IVB & tonsil \\
3 & Male & 60 & yes & T2 N0 M0 & II & tonsil \\
4 & Male & 56 & yes & T3 N2a M0 & IVA & tonsil \\
5 & Male & 52 & yes & T4a N2b M0 & IVA & tonsil \\
6 & Male & 71 & yes & T3 N0 M0 & III & tonsil \\
7 & Male & 66 & yes & T2 N2a M0 & IVA & base of tongue \\
8 & Male & 41 & yes & T3 N1 M0 & III & tongue \\
9 & Male & 42 & Non & T2 N1 M0 & III & tonsil \\
10 & Male & 66 & yes & T4a N1 M0 & IVA & tonsil \\
\hline
\end{tabular}

volume in these patients to which they had to plan a higher dose rate daily than those in conventional treatments (Figure 1).

The variability of the target volume was evident, with relative differences of up to $33 \%$ for the GTV tumor and $94 \%$ for the nodes GTV demonstrating the limited information of the tomography studies when they are used as the only modality in the design of the same being improved with the use of the PET-CT hybrid as shown in Table 2 .

In six of the patients, the clinical-radiological diagnosis was much more extensive than the metabolic labeling and in two lymph node disease was not previously described (Figure 2), allowing us to re-staging them, delineating the volumes to be treated with less extensive margins and performing highly conformal treatments such as intensity modulated radiotherapy (Figure 3).

With the use of PET/CT we were able to obtain high precision in the delivery of radiotherapy, adjusting the prescribed dose to the target volume, decreasing the dose to adjacent healthy tissues as much as possible.

\section{Discussion}

The oncology has experienced in recent years a breakthrough in their treatments and therefore in their results. Similarly, images in general and those modalities used in oncology have also undergone a great development, being able not only to establish a morphological diagnosis, but a functional substrate, both through intrinsic properties of each tissue and the response of these to varied therapies. For example, PET corresponds to a functional image modality whose utility depends on the tracer we use.

Head and neck tumors have been selected as an especially interesting model to detect the value added by PET, given the complexity of their anatomy and the presence of numerous normal structures with variable uptake intensity of FDG $^{18}$ [11].

In theory, PET-FDG can influence the planned volume of radiotherapy 
Table 2. Volume size delineation by CT and PET/CT.

\begin{tabular}{ccccccc}
\hline & \multicolumn{3}{c}{ Volume GTV/Tumor } & \multicolumn{3}{c}{ Volume GTV/Node } \\
\cline { 2 - 7 } Patient & CT & PET/CT & $\begin{array}{c}\text { Relative } \\
\text { Difference }\end{array}$ & CT & PET/CT & $\begin{array}{c}\text { Relative } \\
\text { Difference }\end{array}$ \\
\cline { 2 - 7 } & $\left(\mathrm{cm}^{3}\right)$ & $\left(\mathrm{cm}^{3}\right)$ & $(\%)$ & $\left(\mathrm{cm}^{3}\right)$ & $\left(\mathrm{cm}^{3}\right)$ & $(\%)$ \\
\hline 1 & 25.99 & 22.89 & $13.54 \%$ & 32.24 & 36.68 & $12.12 \%$ \\
2 & 34.00 & 35.75 & $4.88 \%$ & 63.53 & 32.63 & $94.72 \%$ \\
3 & 32.76 & 30.40 & $7.75 \%$ & - & 10.00 & - \\
4 & 43.12 & 32.27 & $33.63 \%$ & 21.76 & 14.33 & $51.83 \%$ \\
5 & 47.02 & 45.62 & $3.06 \%$ & 37.11 & 23.82 & $55.78 \%$ \\
6 & 36.56 & 27.72 & $31.90 \%$ & - & 11.31 & - \\
7 & 37.52 & 35.21 & $6.56 \%$ & 35.01 & 33.79 & $3.60 \%$ \\
8 & 40.29 & 39.86 & $1.08 \%$ & 22.55 & 22.32 & $1.00 \%$ \\
9 & 33.75 & 32.18 & $4.87 \%$ & 15.25 & 18.00 & $15.29 \%$ \\
10 & 48.35 & 44.10 & $9.64 \%$ & 22.90 & 17.59 & $30.18 \%$ \\
\hline
\end{tabular}
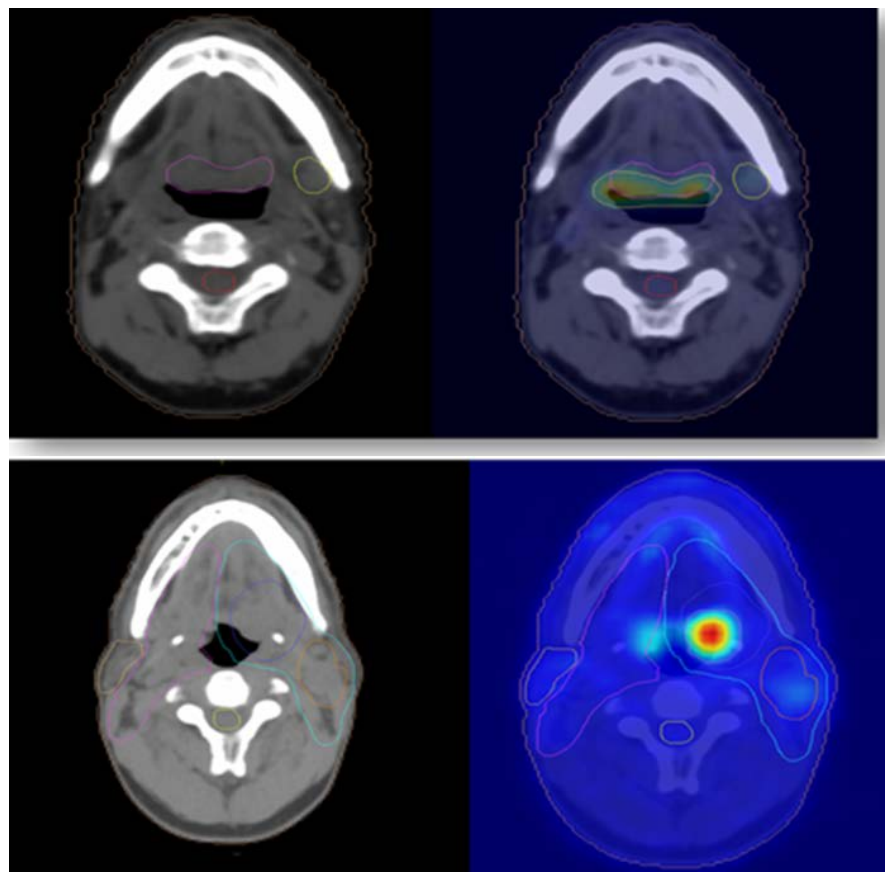

Figure 1. Delineated Volume in Planning CT vs. PET/CT.

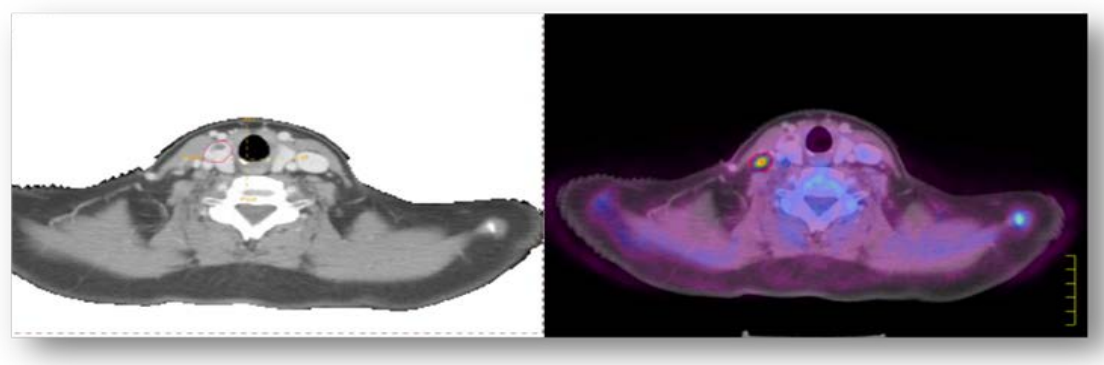

Figure 2. Positive Node identified in the PET image. 

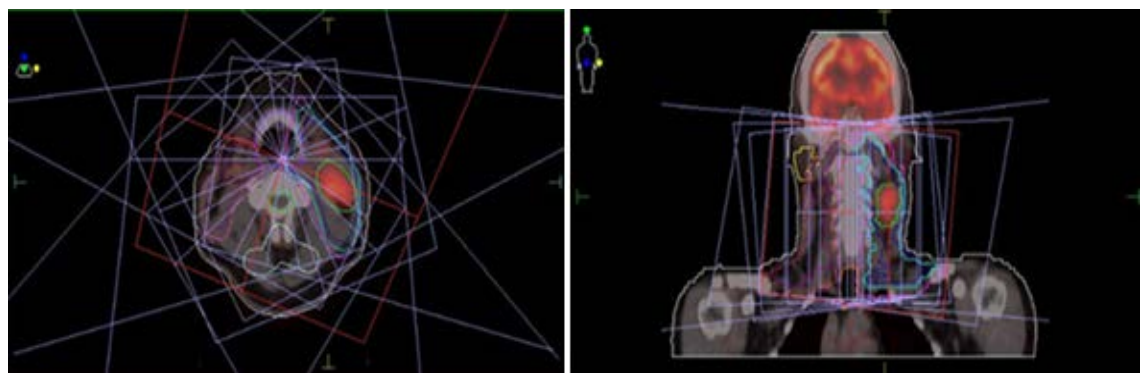

Figure 3. IMRT technique in merged CT and PET/CT image.

treatment and provide a dose reduction on healthy tissue. Facilitating the increase of the dose in the target volume to be treated [12].

According to Daisne et al., the GTV delineated from 18F-FDGPET applying an adaptive signal-to-background method was significantly smaller than GTV delineated by CT or MRI. In addition, GTV-PET was the closest volume to the pathological GTV obtained from surgical specimen. On average, the PET delineated smaller volumes than CT or MRI [13].

Data from the literature reveal an increase in detectable tumor volume (GTV) in up to $20 \%$ of cases if PET/CT is used against CT. Numerous studies evaluate the information provided by PET-FDG in the planning of radiotherapy [14]. As it happened in two of our patients who were detected lymph node disease not previously reported.

In a recent multicentric prospective study by Leclerc et al., the primary tumor was automatically delineated on the 18F-FDG-PET images using a gradient-based method previously described by this group. They confirmed that the use of 18F-FDG-PET smaller GTV, clinical target volume and planning target volume for the primary tumor volumes compared with the use of $\mathrm{CT}$, lowering the dose to organs at risk [15] as it happened in the majority of our patients.

Deniaud-Alexandre et al., in a retrospective study, confirmed that PET-CT has an impact on treatment planning and patient management. In this study, with a total of 101 patients, PET-FDG identified occult metastases in 8 patients (8\%), and GTV decreased, with the fusion of PET-CT images, in 21 patients and increased in 24 patients. The reduction of GTV was $>25 \%$ in 7 patients (7\%) because PET-CT image fusion reduced GTV in 6 patients. The increase in GTV was $>25 \%$ in 14 patients (14\%) due to an increase in GTV in 11 patients [16].

Thus, in Spain, the Health Technology Assessment Agency has carried out an excellent systematic review and a meta-analysis on the indications of the PET-CT. Of 209 articles initially selected, only 16 could be used for the meta-analysis. Of these, 12 were prospective studies and 4 were retrospective, and there was a great variety of types of PET-CT, with different tumors studied and several indications of the procedure. In the specific study, the PET-CT provided an aggregate sensitivity of 0.85 (95\% CI, $0.74-0.92)$ and an aggregate specificity of 0.84 (95\% CI, $0.70-0.93)$. For the tumor re-staging, the sensitivity of the PET-CT was 0.89 (95\% CI, $0.84-0.94)$ and the specificity 0.87 (95\% CI, 0.78 - 
0.93). The authors concluded that this combined technique is a useful technology in tumor staging and re-staging, because it increases the level of confidence in the diagnosis by decreasing the number of equivocal or inconclusive lesions [17].

\section{Conclusions}

18F-FDG PET/CT shows a significant impact on the management of patients with HNSCC on hypofractionated radiotherapy for target volume delineation increasing the visibility, precision in the design and planning in relation to the simple CT as primary imaging modality.

\section{Conflicts of Interest}

The authors declare no conflicts of interest regarding the publication of this paper.

\section{References}

[1] Sigel, R., Naishadham, D. and Jemal, A. (2013) Cancer Statistics 2013. CA: A Cancer Journal for Clinicians, 63, 11-30. https://doi.org/10.3322/caac.21166

[2] Boyle, P. and Levin, B. (2008) World Cancer Report 2008. International Agency for Research on Cancer, Lyon.

[3] NU. CEPAL. Statistics Division (2017) Cuba's Statistical Yearbook for Latinoamerican and the Caribbean. http://interwp.cepal.org/anuario_estadistico/Anuario_2017/index-en.htm

[4] (2017) NCCN Clinical Practice Guidelines in Oncology. http://www.nccn.org/professionals/physician_gls/f_guidelines.asp

[5] (2016) National Cancer Institute Guidelines. https://www.cancer.gov/types/head-and-neck/hp/adult/oropharyngeal-treatment-p dq

[6] Yoo, J., Henderson, S. and Walker-Dilks, C. (2013) Evidence-Based Guideline Recommendations on the Use of Positron Emission Tomography Imaging in Head and Neck Cancer. Clinical Oncology, 25, e33-e66. https://doi.org/10.1016/j.clon.2012.08.007

[7] Eugenio Vinés, V., María José Orellana, G., Catalina Bravo, M. and David Jofré, P. (2017) Manejo del cáncer de cabeza y cuello: ¿Radioterapia a quién, cuándo y por qué? Revista de Otorrinolaringología y Cirugía de Cabeza y Cuello, 77, 81-90. https://doi.org/10.4067/S0718-48162017000100013

[8] Thariat, J., Hannoun-Levi, J.M., Myint, A.S., et al. (2013) Past, Present and Future of Radiotherapy for the Benefitof Patients. Nature Reviews Clinical Oncology, 10, 52-60. https://doi.org/10.1038/nrclinonc.2012.203

[9] Das, S., Thomas, S., Pal, S.K., Isiah, R., John, S., et al. (2013) Hypofractionated Palliative Radiotherapy in Locally Advanced Inoperable Head and Neck Cancer: CMC Vellore Experience. Indian Journal of Palliative Care, 19, 93-98. https://doi.org/10.4103/0973-1075.116709

[10] Grégoire, V., Coche, E., Cosnard, G., Hamoir, M. and Reychler, H. (2000) Selection and Delineation of Lymph Node Target Volumes in Head and Neck Conformal Radiotherapy. Proposal for Standardizing Terminology and Procedure Based on the Surgical Experience. Radiotherapy \& Oncology, 56, 5-50. 
https://doi.org/10.1016/S0167-8140(00)00202-4

[11] Zhu, L. and Wang, N. (2013) 18F-Fluorodeoxyglucose Positron Emission Tomography-Computed Tomography as a Diagnostic Tool in Patients with Cervical Nodal Metastases of Unknown Primary Site: A Meta-Analysis. Surgical Oncology, 22, 190-194. https://doi.org/10.1016/j.suronc.2013.06.002

[12] Cacicedo, J., Navarro, A., Del Hoyo, O., Gomez-Iturriaga, A., Alongi, F., Medina, J.A., et al. (2016) Role of Fluorine-18 Fluorodeoxyglucose PET/CT in Head and Neck Oncology: The Point of View of the Radiation Oncologist. The British Journal of Radiology, 89, Article ID: 20160217. https://doi.org/10.1259/bjr.20160217

[13] Daisne, J.F., Duprez, T., Weynand, B., Lonneux, M., Hamoir, M., Reychler, H., et al. (2004) Tumor Volume in Pharyngolaryngeal Squamous Cell Carcinoma: Comparison at CT, MR Imaging, and FDG PET and Validation with Surgical Specimen. Radiology, 233, 93-100. https://doi.org/10.1148/radiol.2331030660

[14] Geets, X., Lee, J.A., Bol, A., Lonneux, M. and Grégoire, V. (2007) A Gradient-Based Method for Segmenting FDG-PET Images: Methodology and Validation. European Journal of Nuclear Medicine and Molecular Imaging, 34, 1427-1438. https://doi.org/10.1007/s00259-006-0363-4

[15] Leclerc, M., Lartigau, E., Lacornerie, T., Daisne, J.F., Kramar, A. and Gregoire, V. (2015) Primary Tumor Delineation Based on (18) FDG PET for Locally Advanced Head and Neck Cancer Treated by Chemo-Radiotherapy. Radiotherapy \& Oncology, 116, 87-93. https://doi.org/10.1016/j.radonc.2015.06.007

[16] Deantonio, L., Beldi, D., Gambaro, G., Loi, G., Brambilla, M., Inglese, E., et al. (2008) FDG-PET/CT Imaging for Staging and Radiotherapy Treatment Planning of Head and Neckcarcinoma. Radiation Oncology, 3, 29.

https://doi.org/10.1186/1748-717X-3-29

[17] Beswick, D.M., Gooding, W.E., Johnson, J.T. and Branstetter, B.F. (2012) Temporal Patterns of Head and Neck Squamous Cell Carcinoma Recurrence with Positron-Emission Tomography/Computed Tomography Monitoring. Laryngoscope, 122, 1512-1517. https://doi.org/10.1002/lary.23341 